\title{
Observation of Deterministic Chaos in Electrical Discharges in Gases
}

\author{
Thomas Braun, Jorge A. Lisboa, and Ricardo E. Francke \\ Instituto de Física, Universidade Federal do Rio Grande do Sul, 90049 Porto Alegre, Rio Grande do Sul, Brazil \\ and \\ Jason A. C. Gallas \\ Departamento de Física,Universidade Federal de Santa Catarina, 88049 Florianópotis, Santa Catarina, Brazil \\ (Received 12 February 1987)
}

\begin{abstract}
We report that dc-excited discharges in gases display current oscillations which develop into deterministically chaotic behavior. A period-doubling route to chaos characteristic of $1 \mathrm{D}$ maps is established for sealed discharges in Plücker's tubes containing helium. Besides this we report the observation of a scenario showing properties analogous to those of the 2D map of Hénon. Clear sequences of period doublings are also reported for flowing discharges.
\end{abstract}

PACS numbers: $05.45 .+\mathrm{b}, 52.80 .-\mathrm{s}$,

The investigation of nonlinear physical systems exhibiting deterministically chaotic behavior is attracting much attention in recent years. ${ }^{1}$ Among the very interesting nonlinear systems one certainly has gaseous plasmas. Much of the interest in gaseous plasmas derives from their practical applications (e.g., as medium for lasers), their potential applications (e.g., controlled fusion), or the study of their intrinsic properties, ranging from complex plasma structures up to complicated wave-propagation phenomena. As pointed out by Held, Jeffries, and Haller, ${ }^{2}$ although the possibility that plasmas could exhibit deterministically chaotic behavior has been recently discussed theoretically, ${ }^{3,4}$ to date no clear experimental demonstration of such behavior in gaseous plasmas has been given. As far as electrical conduction is concerned, self-generated oscillations have been observed in several substances, ${ }^{5}$ although only a few systems have been studied for deterministically chaotic behavior. ${ }^{6}$ Recently, two interesting results were obtained for solid-state plasmas. ${ }^{2,7}$ As is known, among other uses, semiconductor plasmas are of interest as simple models for some gas plasmas. In the first experiment, Held, Jeffries, and Haller ${ }^{2}$ found chaotic behavior in an electron-hole plasma in a crystal of $\mathrm{Ge}$ under electric and magnetic fields. Martin, Leber, and Martienssen ${ }^{7}$ reported chaotic behavior in the electric conduction of barium sodium niobate single crystals at elevated temperatures. These experiments are particularly beautiful because together with observations made in a $3.39-\mu \mathrm{m}$ $\mathrm{He}-\mathrm{Ne}$ laser by Weiss, Godone, and Olafsson, ${ }^{8}$ they are the first ones to demonstrate a Ruelle-Takens-Newhouse route to chaos outside the domain of hydrodynamics.

In this Letter we report the first observation of deterministically chaotic behavior in a low-energy gaseous plasma, namely in an electric discharge, and show that, depending on control parameters, the current through the discharge as well as the light from the discharge may display stationary, periodic, or chaotic behavior. Further, besides Feigenbaum's well-known period-doubling route to chaos (typical of $1 \mathrm{D}$ maps), we report the observation of a scenario in which a period-doubling cascade is interrupted by the appearence of a bifurcation with a period multiplied by integers other than 2 ( 3 and 5 having the largest basins of attraction). The observation of such a scenario is particularly interesting because of recent work by Libchaber, Fauve, and Laroche ${ }^{9}$ showing that the new scenario might be a true property of $2 \mathrm{D}$ mappings, ${ }^{10}$ instead of a modified or interrupted 1D route. Oscillatory phenomena on dc current applied to a glow discharge has a long history, being first reported more than forty years ago. ${ }^{11}$ These oscillations can be followed by monitoring the voltage, the light emitted, or the current, as we have done here. The main characteristic of the oscillations depends on the particular gas, total pressure, tube diameter, and so on. In spite of being known for more than forty years, no quantitative, nor even a unique qualitative, explanation of these phenomena has been given.

Our experiment is schematically shown in Fig. 1. It consists of measuring the current through sealed electrical discharge tubes of spectral lamps (Plücker's tubes) of the type commonly used to calibrate low-resolution spectra. The nonlinear element in the circuit is the lamp operating in the glow regime, excited by a dc $0-5-\mathrm{kV}$ continuously adjustable high-voltage source. The discharge tube was placed in a water heat bath to stabilize its temperature. $R_{1}$ and $R_{2}$ constitute a $100-\mathrm{k} \Omega$ ballast resistor with $R_{2}$ a $10-\mathrm{k} \Omega$ wire-wound potentiometer used for fine tuning the current. Variations of the current through the lamp were measured from the voltage drop across $R_{3}$ and displayed on the screen of an oscilloscope after conveniently decoupling the dc component by a capacitor $C_{1}$ (see Fig. 1). These variations (typically $0-0.3 \mathrm{~mA}$ in amplitude) were further processed to obtain the trajectory in the phase space $d I / d t \times I$. The dc component of the current was measured by a $3 \frac{1}{2}$-digit multimeter shunted by a capacitor $C_{2}$ as indicated in Fig. 1. The oscillatory phenomena to 


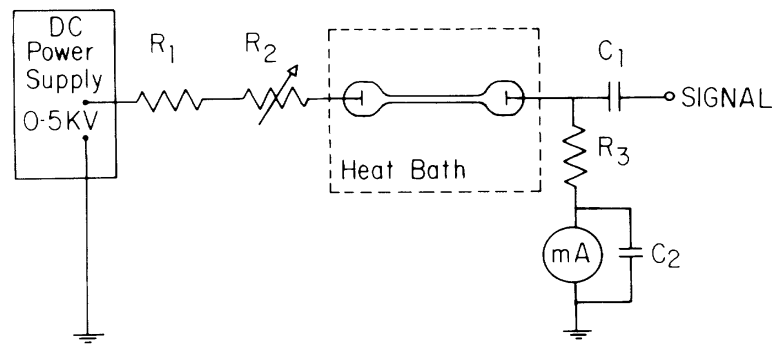

FIG. 1. Schematic diagram of the experiment. $R_{1}=90 \mathrm{k} \Omega$, $R_{2}=10 \mathrm{k} \Omega, R_{3}=5 \mathrm{k} \Omega$, and $C_{1}=C_{2}=0.01 \mu \mathrm{F}$.

be reported below was also observed by monitoring, with a photodiode, the light from the discharge tubes.

The tubes used in our experiments were taken from a CENCO kit designed to serve as reference lamps for teaching purposes. Internal gas pressure and exact diameters of the capilars are unknown. Although all tubes looked very much the same, their behavior under dc excitation was quite different, and (based on experience with flowing tubes to be described below) we attribute this to small differences in the gas pressure. To start, a convenient voltage was applied to the discharge tubes so as to attain a regime with a dc current flowing through the tube. Then, by fine tuning the potentiometer in the circuit we measured the current as described above. Of the tubes at our disposal, those containing argon, neon, and mercury always showed chaotic oscillations, no matter what the current through them. However, in the four helium tubes available, it was possible to see a rich variety of oscillatory behavior, including a Feigenbaum cascade to chaos. The main difference in the behavior of the tubes was the value of the initial current where the period-one oscillations started.

We now describe typical results obtained by measuring the current through the helium discharge of one of the commercial CENCO 87215 spectral lamps (Plücker's tube). In the glow regime, up to $12 \mathrm{~mA}$ one finds just a stationary dc value for the current. Then a self-generated oscillation $f_{0}=190 \mathrm{kHz}$ appears superimposed to the dc level. The left-most trace in Fig. 2(a) shows the time evolution of this oscillation, while the "egg-shaped" graph on the right is the corresponding trajectory in the phase space $\dot{I}(t) \times I(t)$. By gently raising the voltage in the circuit and then tuning $R_{2}$, it is possible to see a very clear period-doubling sequence as displayed in Figs. 2(b)-2(e). By further increasing the current, one observes additional bifurcations, chaos [as shown in Fig. 2(f)], and then an alternation of windows of periodic and chaotic motion following exactly the dynamics predicted for $1 \mathrm{D}$ maps. ${ }^{12}$ It is interesting to mention that the value of the current at bifurcations observed while increasing the current does not exactly correspond to those observed while decreasing the current; i.e., there is some hysteresis involved in the nonlinear processes in the discharge. Furthermore, sometimes dur-

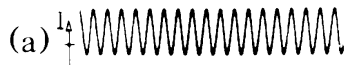
$t+t$

(b) WWWWWWWWW

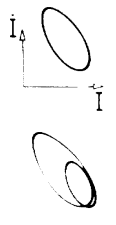

(c) NWWWWWWWWh

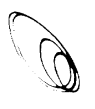

(d)
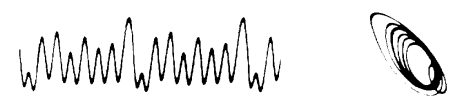

(e)
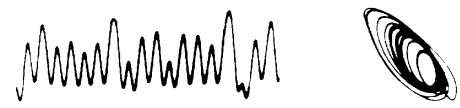

(f)
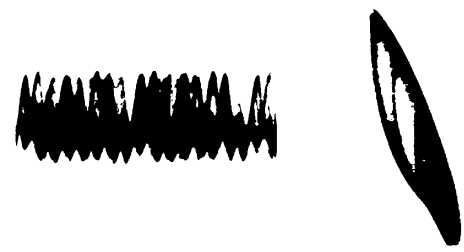

FIG. 2. Experimental results for a helium spectral lamp operating at about $12 \mathrm{~mA}$. Self-generated oscillations of the current $I(t)$ through the lamp are shown on the left with the corresponding phase portraits $d I / d t \times I$ on the right. The current increases from (a) to (f). The scales for $I(t)$ are 0.04 $\mathrm{mA} /$ division and $10 \mu \mathrm{s} /$ division. The phase portrait in ( $\mathrm{f}$ ) is vertically expanded.

ing the bifurcation sequence, we observed the appearence of a low-frequency oscillation superimposed on the signal, in a way similar to that observed by Kitano, Yabuzaki, and Ogawa ${ }^{13}$ while studying bifurcations in an acoustic system. Besides the $1 \mathrm{D}$ route just described, we also observed interruptions of the bifurcation cascade by the sudden appearence of the fundamental frequency followed by further bifurcations. Using results of Libchaber, Fauve, and Laroche ${ }^{9}$ and of Arneodo et al., ${ }^{10}$ we argue below that while 1D maps are not enough to model a discharge, a proper 2D map might be so.

Figure 3 shows what we typically observe in another helium tube operating at higher-current values $\quad(=35$ $\mathrm{mA})$. This figure was obtained by adiabatically decreasing the current through the discharge, starting from a situation corresponding to chaotic oscillation [Fig. 3(e)]. By lowering the current, the first periodic signal seen is the oscillation shown in Fig. 3(d), which repeats itself after forty peaks. By further lowering the current, we enter a region of irregular motion, after which the system starts to oscillate, repeating itself after ten peaks [Fig. 3(c)]. After this, one observes a five-peak periodic oscillation [Fig. 3(b)]. This five-peaked oscillation suffers from the "parasitic" superposition of a low- 
(a) Is

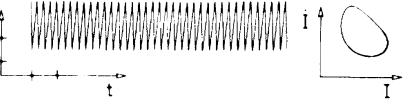

(b)

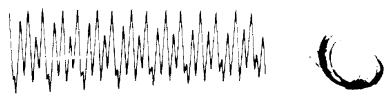

(c)
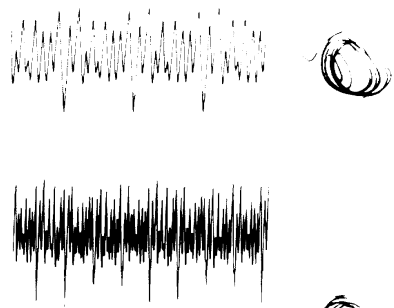

(d)

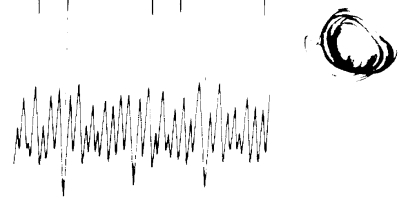

(e)

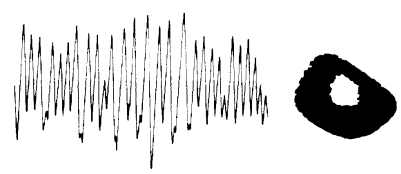

FIG. 3. Experimental results for a helium spectral lamp operating at about $35 \mathrm{~mA}$. The description is similar to that in Fig. 2, the scales being now $0.1 \mathrm{~mA} /$ division and $20 \mu \mathrm{s} /$ division except in graph marked with an asterisk where it is 50 $\mu \mathrm{s} /$ division.

frequency component, as mentioned above. The lowfrequency component tends to make the trajectories in phase space more diffuse. Finally, diminishing the current makes the system oscillate at $f_{0}=190 \mathrm{kHz}$, as shown in Fig. 3(a).

Interesting new aspects of the nonlinear system under study are uncovered if the aforementioned steps are reversed. Starting in (a), an increase in the current produced a period doubling of $f_{0}$, which after destabilization by the low-frequency oscillation turned into an apparently chaotic oscillation. After this, the discharge started to operate in a quite stable manner, showing no parasitic low-frequency interference. By further increasing the current, one reaches the stage (b) which shows a clear period quintupling, similar to the one observed by Linsay $^{14}$ on a driven anharmonic oscillator. From (b) $\rightarrow$ (c) and on the system started a clear sequence of period doublings of the "new" frequency $f_{1}=\frac{1}{5} f_{0}$ and, after several runs of the experiment, it was observed that instead of the irregular gap found before from $(d) \rightarrow(c)$, one now sees the "missing" regular oscillation with twenty peaks and further bifurcations until chaos eventually sets in.

One might think that going from (a) $\rightarrow$ (e) should be equivalent to going from (e) $\rightarrow($ a $)$ and that the reported differences between both paths could be due to not sweeping adiabatically enough the current. Repeated runs of the experiment make us believe that this is not the case. Moreover, to observe the differences, it was much more important that a certain "aging" of the discharge than the actual path followed. In fact, this aging could be perfectly used to classify our tubes as regards to the time they have been effectively used.

Recall that the basic current needed to observe the phenomena described in Fig. 3 (i.e., $35 \mathrm{~mA}$ ) is higher than the corresponding one needed in Fig. 2 (12 mA). The relatively high current level was quite effective in irreversibly changing the characteristics of the discharge in a way that, for example, the aforementioned window between (a) and (b), where it was possible to observe period-two oscillation, diminished with the aging of the tube until the system was apparently jumping directly from period one to chaos. This could be an explanation of the failure to observe bifurcations in our tubes containing argon, neon, and mercury. The aging of the tubes certainly involves electronic processes at the cathode but the exact mechanism at work remains unclear to us. With aging, the current needed to see oscillatory phenomena tended to increase. Besides the period quintupling described above, we also saw period tripling followed by subsequent period doublings.

We also searched oscillatory phenomena in open discharge tubes of the type commonly used in laser spectroscopy of electrically excited flowing gases. The much larger internal diameter of such tubes greatly complicated things. At any rate, it was still possible to see a few bifurcations, but the current tended very much to jump intermittently from periodic to chaotic behavior. Clear, but not easily controllable sequences of bifurcations were observed in helium, argon, $\mathbf{N}_{2}$, and in air. After a selfpulsation had been established in the current, several period doublings were possible to observe by slightly changing the internal gas pressure. We also observed chaos through the same sequence of period doublings described before by directly changing the impedance of the Plücker discharges. This was achieved by sweeping a variable capacitor grounded through a piece of wire hanging directly over the capillary tube. A further interesting point is that placing the discharge tube inside a heat bath of water had the desirable effect of enhancing the stability of the oscillations. Without bath, the heating caused by increments in the current was sometimes able to induce new bifurcations. In fact, with no changes on the electric circuit it was possible to generate sequences of period doublings by simple heating or cooling the bath. The current needed to observe the self-induced oscillations increased with the temperature of the bath.

A few words about the theory of gas discharges are in order. A microscopic description of them involves distribution functions and the Boltzmann equation. To obtain the current, however, just average values of quantities 
such as number density, velocity, and energy are needed. This is essentially the macroscopic point of view, based on the fluid, or moment, equation obtained from appropriate averages over the Boltzmann equation. The plasma acts as a conducting fluid and, indeed, many of the basic properties of gas discharges can be explained on the basis of fluid equations. ${ }^{15}$ The current nev is controlled by

$$
\partial n / \partial t+\nabla \cdot n v=\alpha n-\beta n^{2},
$$

where $n$ is the density of electrons, $v$ the drift velocity, and $\alpha$ and $\beta$ are ionization and recombination coefficients, respectively. The drift velocity depends on the electric field according to

$$
n v=-\frac{n e}{m} \gamma_{m} E-\frac{k T}{m} \gamma_{m} \nabla n,
$$

$\gamma_{m}$ being the electron-atom collision frequency and $e, m$, $k$, and $T$ having their usual meaning. The electric field $E$ should be obtained from Maxwell's equations. Thus, the above equations coupled to the Maxwell equations constitute a set of nonlinear partial differential equations (PDE) describing our experiment. The investigation of PDE for possible chaotic behavior is still in the beginning stage. ${ }^{16}$ The usual approach is to truncate them into a set of ordinary equations. The paradigm of chaotic motion, the Lorenz model, is obtained by this procedure from PDE describing a fluid between parallel plates. It is therefore plausible to expect the above set of PDE to display chaos. On the other hand, we would like to mention recent work ${ }^{9,10}$ where the puzzling result of a period-doubling cascade interrupted by odd-period multiplication (not consistent with predictions from 1D maps, even if fluctuations and nonadiabaticity in control parameters are considered) was shown to occur in an invertible $2 \mathrm{D}$ map (Hénon map) if one allows nonadiabatic changes in control parameters. The competition between period doubling and period multiplication by odd integers is exactly what we observe in discharges. Therefore, eventually "ordinarizations" of the above PDE are expected to show a richer (i.e., 2D) Poincaré map than the usual $1 \mathrm{D}$ result, typical of Feigenbaum cascades.

In conclusion, we reported deterministic chaos to occur in dc-excited electrical discharges in gases. A Feigenbaum cascade was shown to exist in sealed capillary discharges through helium, and sequences of period doublings were reported to occur in flowing systems. The phenomena were shown to depend on a large number of variables. Although a quantitative and even a unique qualitative description of the mechanism responsible for the appearance of oscillatory behavior was and still is lacking, it was pointed out that chaos is to be expected from a macroscopic description of the discharge and that the system, in spite of having many degrees of freedom, can be modeled by $1 \mathrm{D}$ maps. Further, compet- ing with the Feigenbaum cascade, we reported what appears to be the second experimental example of a scenario seen on the 2D map of Hénon. Thus, even though the discharge involves many degrees of freedom, it shows complicated behavior that is similar to those present in $1 \mathrm{D}$ and $2 \mathrm{D}$ maps. It is remarkable that these mathematical entities can provide such a degree of analogy with the dynamics of nonequilibrium physical systems with many degrees of freedom.

One of us (J.A.C.G.) is a research fellow of the Brazilian Research Council (Conselho Nacional de Pesquisas).

\footnotetext{
${ }^{1}$ Besides almost every issue of, for example, Phys. Rev. Lett. over the last few years, see the review of H. L. Swinney, Physica (Utrecht) 7D, 3 (1983); J. D. Farmer, in Evolution of Order and Chaos, edited by H. Haken (Springer-Verlag, New York, 1982).

${ }^{2}$ G. A. Held, C. Jeffries, and E. E. Haller, Phys. Rev. Lett. 52, 1037 (1984).

${ }^{3}$ J.-M. Wersinger, J. M. Finn, and E. Ott, Phys. Fluids 23, $1142(1980)$.

${ }^{4}$ J. C. Adam, M. N. Bussac, and G. Laval, in Intrinsic Stochasticity in Plasmas, edited by G. Laval and D. Grésillon (les Edition de Physique, Orsay, 1979).

${ }^{5}$ J. Keizer, in Special Topics in Electrochemistry, edited by P. A. Rock (Elsevier, Amsterdam, 1977); F. Kaiser, Z. Naturforsch. 33A, 294, 418 (1978); F. Stöckmann, in Festkörperprobleme: Advances in Solid State Physics, edited by O. Madelung (Vieweg, Braunschweig, 1969), Vol. 9; J. B. Gunn, Solid State Commun. 1, 88 (1963); V. V. Voronov and Yu. S. Kuz'minov, Fiz. Tverd. Tela (Leningrad) 20, 389 (1978) [Sov. Phys. Solid State 20, 224 (1984)]; for further references see Ref. 2.

${ }^{6}$ R. M. Fleming and C. C. Grimes, Phys. Rev. Lett. 42, 1423 (1979); K. Aoki, K. Miyamae, T. Kobayashi, and K. Yamamoto, Physica (Utrecht) B\&C 117\& 118, 570 (1983).

${ }^{7}$ S. Martin, H. Leber, and W. Martienssen, Phys. Rev. Lett. 53, 303 (1984).

${ }^{8}$ C. O. Weiss, A. Godone, and A. Olafsson, Phys. Rev. A 28, 892 (1983)

${ }^{9}$ A. Libchaber, S. Fauve, and C. Laroche, Physica (Utrecht) 7D, 73 (1983).

${ }^{10}$ A. Arneodo et al., Physica (Utrecht) 6D, 385 (1983).

${ }^{11}$ B. Granovsky and L. Bykhovskaya, J. Phys. (Moscow) 10, 351 (1946); T. Donahue and G. H. Dieke, Phys. Rev. 81, 248 (1951); for a review, see G. Francis, in Gasentladungen II, Handbuch der Physik, Band 22, edited by S. Flugge (Springer-Verlag, Berlin, 1957).

$12 \mathrm{~J}$. Collet and J. P. Eckmann, Iterated Maps of the Interial as Dynamical Systems (Birkhäuser, Boston, 1980).

${ }^{13}$ M. Kitano, T. Yabuzaki, and T. Ogawa, Phys. Rev. Lett. 50, 713 (1983)

${ }^{14}$ P. Linsay, Phys. Rev. Lett. 47, 1349 (1981).

${ }^{15}$ B. E. Cherrington, Gaseous Electronics and Gas Lasers (Pergamon, Oxford, 1979).

${ }^{16}$ D. R. Moore et al., Nature (London) 303, 663 (1983).
} 